\title{
Cash and livelihoods in contexts of conflict and fragility
}

\author{
Jeremy Lind, Rachel Sabates-Wheeler and Carolina Szyp
}

February 2022

\section{Theme summary}

Multiple efforts have been made in recent years to introduce cash transfers augmented by livelihood support ('cash-plus') into protracted crisis contexts to support lives and livelihoods. Yet, little learning has been generated about how to design and implement these effectively and under what conditions.

This brief summarises the state of the evidence and debate, gaps in the evidence, and directions for research that emerge from the thematic paper on cash-plus in protracted crises characterised by conflict and fragility. We identify distinct objectives and patterns of cash-plus provision across different conflict-social protection contexts. A dearth of evidence on impacts of these programmes remains. This has implications for future work in the area of cash assistance and livelihoods in protracted crisis settings. We identify a number of key questions to guide further research.

This thematic brief is a shortened version of a BASIC Research Working Paper. To explore this research theme in more detail please refer to:

Lind, J.; Sabates-Wheeler, R. and Szyp, C. (2022) Cash and Livelihoods in Contexts of Conflict and Fragility: Implications for Social Assistance Programming, BASIC Research Working Paper 8, Brighton: Institute of Development Studies, DOI: 10.19088/BASIC.2022.008

A full list of the references cited in this brief can be found at the BASIC Research Zotero library.

Implemented by

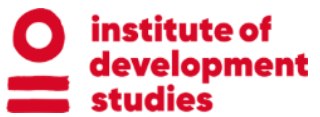

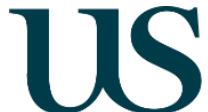

UNIVERSITY OF SUSSEX
UNIVERSITY OF WOLVERHAMPTON

Better Assistance in Crises (BASIC) Research (funded by UKAid) aims to inform policy and programming on how to help poor and vulnerable people cope better with crises and meet their basic needs through more effective social assistance. All costs related to BASIC Research are covered by the UK Foreign, Commonwealth and Development Office. 


\section{State of the evidence and debate}

A vision for resilience and long-term sustainability needs to inform how and under what conditions conflict and crisis-affected populations can secure their livelihoods. To this end, there have been multiple recent efforts to experiment with cash-plus approaches. The assumption is that cash augmented with other forms of support (components and linkages) will, over time, contribute to creating 'productive' and/or 'sustainable' livelihoods for beneficiaries, even once social assistance is withdrawn (Roelen et al. 2017). However, evidence on the impacts and outcomes of such approaches in promoting and transforming livelihoods is lacking, especially regarding crisis and conflict areas.

Cash-plus provision is primarily tailored for households and individuals; little attention is paid to the broader economic, political and security contexts within which people live and work. Whether programmes are successful, and whether they are even an option, depends on a wider environment (including available infrastructure, physical security, accessible markets). The strength and maturity of the social protection system affects the design and implementation of social assistance - begging the question: how to strengthen livelihoods in protracted crises, particularly when types of violence and armed activity are aimed at destroying livelihoods or when state infrastructure is weak or non-existent.

The intensity of conflict (a defining feature of many protracted crises) can be considered alongside the strength of state-led social protection systems, to develop broad categories describing contexts for cashplus programming (see figure). At one extreme - in settings with no armed conflict-related fatalities, but with existing social protection (green quadrant) - it should be possible to provide the full range of services in parallel to supporting improvements in livelihoods along a pathway to graduation. At the other extreme (red quadrant), the challenges for cash-plus programming are greatest in areas that experience more intense conflict, during periods when conflict levels are acutely high, and where social protection systems and programmes are shattered or non-existent.

Figure 1: Implementation contexts for cash-plus programming in protracted crises characterised by conflict

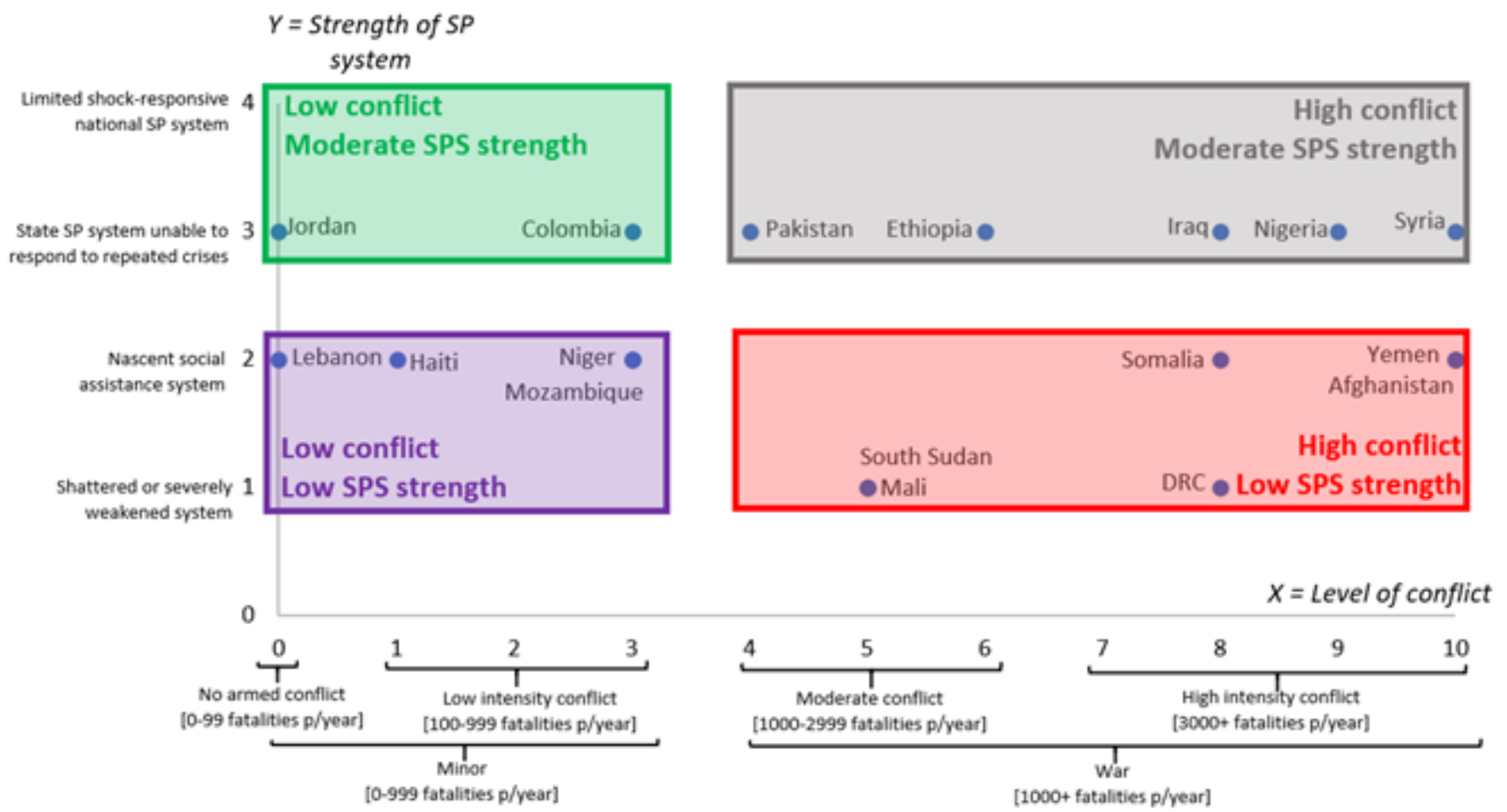

Source: Authors' own 


\section{Gaps in the evidence}

We reviewed 42 cash-plus programmes in 17 countries with protracted crises ${ }^{1}$ to determine whether clear differences exist in the provision methods and types of complementary support, and to gauge whether these cash-plus programmes are fit for conflict-affected settings.

\section{Key findings:}

- Most programmes combine design features seen in more peaceful contexts - skills being the most common component, followed by agricultural and livestock extension services and access to finance. In settings with moderately strong social protection systems, government agencies and sub-national public administration are by far the most common implementers. In areas with higher conflict levels, NGOs - international NGOs in particular - dominate this role. Programmes are often small scale in lower-conflict areas and larger-scale in higher-conflict areas, thus, conflictrelated violence should not impede cash-plus programming.

- The most common objective of cash-plus programmes is 'promotion', closely followed by relief (or protection), particularly in areas experiencing higher levels of conflict-related violence. Relatively few programmes incorporate objectives around resilience, social cohesion or mitigating the factors contributing to conflict. Less than a quarter of programmes in high-conflict areas mention peace-building objectives.

- Evaluation of social assistance livelihoods programmes is very patchy and mostly focused on short-term impacts. Some programmes refer to the number of community assets that were constructed (outputs), with vague reference to impacts or outcomes indicators, including costeffectiveness. The majority of assessments occurred at the endline or shortly after the end of the programme (typically within two years). Assessments of performance rely heavily on the statements of implementing agencies and the donors that fund them. Rigorous and impartial evidence is limited.

1 These include Yemen, Somalia, Lebanon, Jordan, Niger, Mali, Nigeria, Iraq, Democratic Republic of the Congo, Congo, South Sudan, Ethiopia, Mozambique, Central African Republic, Libya, Syria, Afghanistan, Myanmar, Pakistan, Haiti, Colombia, Venezuela, Ukraine. These countries are the focus of the BASIC Research programme and were selected as contexts where there are overlapping challenges from protracted conflict, recurrent climate shocks, and protracted displacement. 


\section{Directions for research}

Our findings suggest that cash-plus components can and should be tailored to suit contexts defined by the intensity of conflict and the strength (or maturity) of the existing social protection system. Research must identify how cash-plus models can be adapted in crises and which are most effective at strengthening livelihoods. This should contribute to a greater objective of empowerment of those most excluded, and to structural transformation that supports livelihood resilience.

\section{Research aims:}

- More comprehensively assess the design and implementation features of cash-plus programmes in protracted crises and generate evidence of the relative effectiveness of different approaches. Research could discern which modifications, if any, are most likely to increase programme effectiveness in settings of protracted conflict and displacement, and climate shocks.

- Critically reflect on ways of linking cash-plus programmes implemented by humanitarian actors with state social protection systems and programmes, to expand the scale and reach of interventions that demonstrate effectiveness.

- Examine the potential contribution of social assistance and livelihoods programming to broader efforts to prevent conflict and promote social cohesion. Include the extent to which conflict prevention and violence mitigation should be incorporated within (or kept separate from) objectives, modalities, and targeting criteria of cash-plus programmes.

\section{An overarching question emerges:}

- How can social assistance best contribute to livelihoods that show greater resilience or adaptation to protracted conflict, climate shocks and forced displacement?

\section{Sub-questions:}

- How are cash-plus models adapted in crises?

- What is the evidence of outcomes and impact and how does context mediate these outcomes? What does the evidence imply for how cash-plus models need to be adapted in crisis contexts?

- How can NGO/UN agency-implemented programmes work with governments to build social protection systems, and under what conditions?

- Are these programmes able to cater for the different drivers of vulnerability within their design and implementation, across contexts characterised by multiple, intersecting drivers of vulnerability.

\section{Acknowledgements and Disclaimer}

This document was developed by the Better Assistance in Crises (BASIC) Research programme. BASIC is implemented by the Institute of Development Studies (IDS), the University of Sussex and the Centre for International Development and Training, funded by UKAid from the UK government. The views expressed in this document are entirely those of the authors and do not necessarily represent views or policies of the UK governments official policies.

(C) IDS copyright 2022. Copyright in the typographical arrangement and design rests with IDS.

This publication (excluding the logos) may be reproduced free of charge in any format or medium, provided that it is reproduced accurately and not used in a misleading context. The material must be acknowledged as IDS copyright with the title and source of the publication specified.

Published by IDS.

DOI: $10.19088 / B A S I C .2022 .028$ 